\title{
Time-optimal control of a 3-level quantum system and its generalization to an $n$-level system
}

\author{
Dong Eui Chang and Rodolphe Sepulchre
}

\begin{abstract}
We solve the problem of steering a three-level quantum system from one eigen-state to another in minimum time and study its possible extension to the time-optimal control problem for a general $n$-level quantum system. For the threelevel system we find all optimal controls by finding two types of symmetry in the problem: $\mathbb{Z}_{2} \times S_{3}$ discrete symmetry and $S^{1}$ continuous symmetry, and exploiting them to solve the problem through discrete reduction and symplectic reduction. We then study the geometry, in the same framework, which occurs in the time-optimal control of a general $n$-level quantum system.
\end{abstract}

\section{The Statement of the Problem}

In this paper we study the time-optimal control problem for the following 3-level system:

$$
\left\{\begin{array}{l}
\dot{x}_{1}=-\omega_{3} x_{2} \\
\dot{x}_{2}=\omega_{3} x_{1}-\omega_{1} x_{3} \\
\dot{x}_{3}=\omega_{1} x_{2}
\end{array}\right.
$$

with the initial and final conditions

$$
\mathbf{x}(0)=(1,0,0), \quad \mathbf{x}\left(T_{\min }\right)=(0,0,1)
$$

and the control constraints

$$
\left|\omega_{1}\right| \leq 1, \quad\left|\omega_{3}\right| \leq 1
$$

We show that there are exactly two optimal control laws:

$$
\left(\omega_{1}, \omega_{3}\right)= \pm(1,1)
$$

and the minimum time cost is $\frac{\pi}{\sqrt{2}}$. Furthermore, we show how the same technique can be extended to understanding the geometry in the time-optimal control problem for the general $n$-level system:

$$
\left\{\begin{array}{l}
\dot{x}_{1}=-u_{1} x_{2} \\
\dot{x}_{2}=u_{1} x_{1}-u_{2} x_{3} \\
\quad \vdots \\
\dot{x}_{n-1}=u_{n-2} x_{n-2}-u_{n-1} x_{n} \\
\dot{x}_{n}=u_{n-1} x_{n-1}
\end{array}\right.
$$

with the initial and final conditions

$$
\mathbf{x}(0)=(1,0, \cdots, 0), \quad \mathbf{x}\left(T_{\min }\right)=(0, \cdots, 0,1)
$$

and the control constraints

$$
\left|u_{i}\right| \leq 1, \quad i=1, \ldots, n-1
$$

D.E. Chang is with the Department of Applied Mathematics, University of Waterloo, Waterloo, ON N2L 3G, Canada dechang@math. uwaterloo. ca

R. Seplchre is with the Department of Electrical Engineering and Computer Science, University of Liège, B-4000 Liège Sart-Tilman,Belgium r.sepulchredulg.ac.be
In the context of quantum mechanics, the model considered in this paper is a finite-dimensional low-energy approximation of a Schrödinger equation driven by rotating fields and averaged over a time interval longer than the inverse energy splittings, where each $x_{i}$ corresponds to the coefficient of the eigen wave function of the $i$-th energy level, and controls $u_{i}$ 's correspond to the amplitudes of lasers [11], [5].

Various open-loop control problems for quantum systems have been already studied. In particular, the energy-optimal control problem for the dynamics in (4) without any magnitude constraints on control was studied at the level of Lie groups in [7], [4]. For that problem, the author in [7] combined Lie-Poisson reduction theory with the Pontryagin Maximum Principle (PMP), and the authors in [4] utilized sub-Riemannian geometry with the PMP. In [11], the trajectory generation problem for the dynamics was studied via flatness theory. In [3] the time-optimal control problem for the dynamics in (1)-(3) using sub-Riemmanian geometry with the PMP. The same problem and its generalization are studied in this article using a different approach. Our main tool, distinct from those in [3], [4], [6], [7], [11], is the detection and exploitation of both continuous and discrete symmetry in the problem. An example of this is an $S^{1}$ continuous symmetry and a $\mathbb{Z}_{2} \times S_{3}$ discrete symmetry in the dynamics (1)-(3). We employ discrete reduction and symplectic reduction theory to remove those symmetries and simplify the dynamics. This technique can also be effectively generalized to the time-optimal control of a general $n$-level quantum system.

\section{Pontryagin MaXimum Principle}

We review the Pontryagin Maximum Principle for timeoptimal control problems. Consider a control system

$$
\dot{x}=f(x, u), \quad(x, u) \in \mathbb{R}^{n} \times U
$$

where $U$ is a compact subset of $\mathbb{R}^{k}$. Define a Hamiltonian function on $\mathbb{R}^{n} \times \mathbb{R}^{n} \times U$

$$
H(x, p, u)=\langle p, f(x, u)\rangle
$$

where $p \in \mathbb{R}^{n}$ is a covector. Then the following holds:

Theorem II.1. ([9]) Let $u(t)$ be a time-optimal control on $\left[0, T_{\min }\right]$ for the system (7) with the boundary conditions

$$
x(0) \in N_{0}, \quad x\left(T_{\min }\right) \in N_{1}
$$

where $N_{0}$ and $N_{1}$ are regular submanifolds of $\mathbb{R}^{n}$. Let $x(t)$ be the corresponding optimal trajectory. Then, there exists 
a nonzero continuous covector function $p(t) \in \mathbb{R}^{n}$ such that $(x(t), p(t), u(t))$ satisfies

$$
\dot{x}=\frac{\partial H}{\partial p}, \quad \dot{p}=-\frac{\partial H}{\partial x}
$$

with $H$ in (8), where

1. $u(t)=\arg \sup _{v \in U} H(x(t), p(t), v) \forall t \in\left[0, T_{\min }\right]$.

2. $H(x(t), p(t), u(t))=M(x(t), p(t))$ almost everywhere in $\left[0, t_{f}\right]$ where $M(x, p)=\sup _{v \in U} H(x, p, v)$.

3. $M(x(t), p(t))=$ constant on $\left[0, T_{\min }\right]$.

4. $\left\langle p(0), T_{x(0)} N_{0}\right\rangle=0,\left\langle p\left(T_{\min }\right), T_{x\left(T_{\min }\right)} N_{1}\right\rangle=0$.

Corollary II.2. Suppose that the boundary conditions in (9) are fixed points as follows: $x(0)=x_{0}, \quad x\left(T_{\min }\right)=x_{1}$, and that there exists a regular submanifold $L \subset \mathbb{R}^{n}$ containing all trajectories of (7) reaching $x_{1}$. Then, $p(0) \in T_{x_{0}} L \subset \mathbb{R}^{n}$.

\section{Time-Optimal Control OF The ThreE-LEVEL QUANTUM SYSTEM}

It is straightforward to see that our optimal control problem satisfies the conditions in Theorem 4 in $\S 4.4$ of [8]. Hence, there exist time-optimal trajectories for our system with the minimum time cost $T_{\min }$.

a) Discrete Symmetry: We study the discrete symmetry in the system. For brevity, we write (1) in compact form as follows:

$$
\dot{\mathbf{x}}=A\left(\omega_{1}, \omega_{3}\right) \mathbf{x}
$$

where

$$
A\left(\omega_{1}, \omega_{3}\right)=\left[\begin{array}{ccc}
0 & -\omega_{3} & 0 \\
\omega_{3} & 0 & -\omega_{1} \\
0 & \omega_{1} & 0
\end{array}\right] .
$$

Let $g_{1}, g_{2}$ and $g_{3}$ respectively, be the reflection in the plane $P_{1}=\left\{x_{1}=0\right\}, P_{2}=\left\{x_{2}=0\right\}$ and $P_{3}=\left\{x_{3}=0\right\}$ respectively. They are given in matrix form by

$g_{1}=\operatorname{diag}(-1,1,1), g_{2}=\operatorname{diag}(1,-1,1), g_{3}=\operatorname{diag}(1,1,-1)$

We claim that the system in (1) with (3) is invariant under $g_{1}, g_{2}$ and $g_{3}$. For example, notice that

$$
\left(g_{2}\right)^{-1} A\left(\omega_{1}, \omega_{3}\right) g_{2}=A\left(-\omega_{1},-\omega_{3}\right) .
$$

Suppose that there is a control $\left(\omega_{1}(t), \omega_{3}(t)\right)$ on the time interval $[0, T]$ and there exists a sub-interval $\left[t_{1}, t_{2}\right] \subset[0, T]$ such that the trajectory $\mathbf{x}(t)=\left(x_{1}(t), x_{2}(t), x_{3}(t)\right)$ driven by the control satisfies

$$
x_{2}\left(t_{1}\right)=x_{2}\left(t_{2}\right)=0, \quad \text { and } \quad x_{2}(t)<0 \quad \text { for } t \in\left(t_{1}, t_{2}\right) .
$$

If the following control

$$
\left(\tilde{\omega}_{1}(t), \tilde{\omega}_{3}(t)\right)= \begin{cases}\left(\omega_{1}(t), \omega_{3}(t)\right) & \text { for } 0 \leq t \leq t_{1}, \\ \left(-\omega_{1}(t),-\omega_{3}(t)\right) & \text { for } t_{1}<t<t_{2}, \\ \left(\omega_{1}(t), \omega_{3}(t)\right) & \text { for } t_{2} \leq t \leq T\end{cases}
$$

is used, then the associated trajectory $\tilde{\mathbf{x}}(t)=$ $\left(\tilde{x}_{1}(t), \tilde{x}_{2}(t), \tilde{x}_{3}(t)\right)$ will satisfy

$$
\tilde{\mathbf{x}}(t)= \begin{cases}\left(x_{1}(t), x_{2}(t), x_{3}(t)\right) & \text { for } 0 \leq t \leq t_{1} \\ \left(x_{1}(t),-x_{2}(t), x_{3}(t)\right) & \text { for } t_{1}<t<t_{2} \\ \left(x_{1}(t), x_{2}(t), x_{3}(t)\right) & \text { for } t_{2} \leq t \leq T\end{cases}
$$

In particular,

$$
\tilde{x}_{2}\left(t_{1}\right)=\tilde{x}_{2}\left(t_{2}\right)=0 \quad \text { and } \quad \tilde{x}_{2}(t)>0 \quad \text { for } t \in\left(t_{1}, t_{2}\right) .
$$

Notice that $\mathbf{x}(0)=\tilde{\mathbf{x}}(0), \mathbf{x}(T)=\tilde{\mathbf{x}}(T)$, and that the trajectory $\tilde{\mathbf{x}}(t)$ has the same time cost $T$. Hence, there always exists a time-optimal trajectory contained in the set $\left\{x_{2} \geq 0\right\}$. By applying similar arguments to $g_{1}$ and $g_{3}$, the following lemma can be deduced:

Lemma III.1. There exists a time-optimal trajectory contained in the closure $\overline{\mathcal{O}}_{1}$ of the first (open) octant

$$
\mathcal{O}_{1}=\left\{\left(x_{1}, x_{2}, x_{3}\right) \in \mathbb{R}^{3} \mid x_{1}>0, x_{2}>0, x_{3}>0\right\} .
$$

We now consider the reflection $g_{4}$ in the plane

$$
\Pi=\left\{x_{1}=x_{3}\right\},
$$

where $g_{4}$ is given in matrix form by

$$
g_{4}=\left[\begin{array}{lll}
0 & 0 & 1 \\
0 & 1 & 0 \\
1 & 0 & 0
\end{array}\right]
$$

Notice that

$$
\left(g_{4}\right)^{-1} A\left(\omega_{1}, \omega_{3}\right) g_{4}=A\left(\omega_{3},-\omega_{1}\right)
$$

Since the hyperplane $\Pi$ divides $\mathbb{R}^{3}$ into two regions such that $\mathbf{x}(0)=(1,0,0)$ and $\mathbf{x}\left(T_{\min }\right)=(0,0,1)$ belong in distinct regions, every trajectory from $(1,0,0)$ to $(0,0,1)$ must intersect with $\Pi$. Suppose that there is a control $\left(\omega_{1}, \omega_{3}\right):[0, T] \rightarrow[-1,1]^{2}$ for (1) such that the associated trajectory $\left(x_{1}(t), x_{2}(t), x_{3}(t)\right)$ with the initial condition $(1,0,0)$ reaches $\Pi$ at $t=T$ for the first time. We extend the control to the time interval $[0,2 T]$ as follows:

$$
\omega_{1}(T+s)=\omega_{3}(T-s), \omega_{3}(T+s)=\omega_{1}(T-s)
$$

for $s \in[0, T]$. By (12) and the consideration of time-reversal, $\mathbf{x}(t)$ on $[0,2 T]$ satisfies

$$
g_{4}(\mathbf{x}(T-s))=\mathbf{x}(T+s), \quad s \in[0, T] .
$$

Hence, the trajectory $\mathbf{x}(t)$ for $t \in[0,2 T]$ is invariant under the reflection with respect to the plane $\Pi$, and thus $\mathbf{x}(2 T)=$ $(0,0,1)$. This observation leads us to the following lemma:

Lemma III.2. Consider the time-optimal control problem for the system (1) with (2) and (3). Then the following holds.

1. There exists a time-optimal trajectory which is symmetric with respect to the plane $\Pi$.

2. Every time-optimal trajectory reaches $\Pi$ in minimum time, which is half of the total minimum time cost.

3. Every time-optimal trajectory intersects with $\Pi$ only once. As a result, there is no segment in any optimal trajectory which totally lies in $\Pi$.

Lemma III.3. Consider the time-optimal control problems for the system (1) with the constraint (3) and the following eight distinct initial and final conditions: 


\begin{tabular}{|c|c|c|c|}
\hline $\mathbf{x}_{0}$ & $\mathbf{x}_{1}$ & $\mathbf{x}_{0}$ & $\mathbf{x}_{1}$ \\
\hline$(1,0,0)$ & $(0,0,1)$ & $(0,0,1)$ & $(1,0,0)$ \\
\hline$(1,0,0)$ & $(0,0,-1)$ & $(0,0,1)$ & $(-1,0,0)$ \\
\hline$(-1,0,0)$ & $(0,0,1)$ & $(0,0,-1)$ & $(1,0,0)$ \\
\hline$(-1,0,0)$ & $(0,0,-1)$ & $(0,0,-1)$ & $(-1,0,0)$ \\
\hline
\end{tabular}

Then, they all have the same minimum time costs.

We note that the group generated by $\left\{g_{i} \mid i=1,2,3,4\right\}$ is isomorphic to $\mathbb{Z}_{2} \times S_{3}$ where $S_{3}$ is the symmetric group on 3 letters.

b) Maximum Principle: By Lemma III.1 we will initially look for all time-optimal trajectories which are contained in $\overline{\mathcal{O}}_{1}$, i.e.,

$$
\mathbf{x}\left(\left[0, T_{\min }\right]\right) \subset \overline{\mathcal{O}}_{1}
$$

However, it is important to notice that this does not impose any state constraints on our optimal control problem. Hence, we can apply the ordinary Pontryagin Maximum Principle, which does not take into account any state constraints, to the system (1) - (3) satisfying (14).

Following (8), we construct the Hamiltonian

$$
H=\omega_{1}\left(x_{2} p_{3}-x_{3} p_{2}\right)+\omega_{3}\left(x_{1} p_{2}-x_{2} p_{1}\right)
$$

where $\mathbf{p}=\left(p_{1}, p_{2}, p_{3}\right)$ is a nonzero covector satisfying

$$
\dot{\mathbf{p}}=A\left(\omega_{1}, \omega_{3}\right) \mathbf{p}
$$

with $A\left(\omega_{1}, \omega_{3}\right)$ in (11). The optimal control satisfies

$$
\left\{\begin{array}{l}
\omega_{1}(t)=\operatorname{sign}\left(x_{2}(t) p_{3}(t)-x_{3}(t) p_{2}(t)\right), \\
\omega_{3}(t)=\operatorname{sign}\left(x_{1}(t) p_{2}(t)-x_{2}(t) p_{1}(t)\right)
\end{array}\right.
$$

where it is assumed that the sign function at 0 can take an arbitrary value between -1 and 1 . By the third statement of Theorem II.1, we have

$$
M(\mathbf{x}, \mathbf{p})=\left|x_{2} p_{3}-x_{3} p_{2}\right|+\left|x_{1} p_{2}-x_{2} p_{1}\right|
$$

and

$$
M(\mathbf{x}(t), \mathbf{p}(t))=M(\mathbf{x}(0), \mathbf{p}(0))
$$

for $t \in\left[0, T_{\min }\right]$ along each optimal trajectory $(\mathbf{x}(t), \mathbf{p}(t))$.

Since the vector field (10) at $\mathrm{x} \in \mathbb{R}^{3}$ is orthogonal to $\mathbf{x}$ and the initial and final points in (2) belong to the unit 2-sphere

$$
S^{2}=\left\{\mathbf{x} \in \mathbb{R}^{3} \mid\|\mathbf{x}\|=1\right\},
$$

the time-optimal control problem is essentially defined on $S^{2}$. By Corollary II.2 we have the following transversality condition at $t=0$ :

$$
p_{1}(0)=0 .
$$

Since $\mathbf{p}(0) \neq 0$ by the Maximum Principle and $\|\mathbf{p}(t)\|=$ $\|\mathbf{p}(0)\| \neq 0$ by (16), the $\mathbf{p}$-dynamics is defined on $\mathbb{R}^{3}-$ $\{(0,0,0)\}$. From (10) and (16), it follows that $\frac{d}{d t}\langle\mathbf{x}, \mathbf{p}\rangle=0$. Hence,

$$
\langle\mathbf{x}(t), \mathbf{p}(t)\rangle=\langle\mathbf{x}(0), \mathbf{p}(0)\rangle=0
$$

where (2) and (20) were used. Therefore, the (x, p)dynamics in (10) and (16) are defined on

$$
P=\left\{(\mathbf{x}, \mathbf{p}) \in \mathbb{R}^{3} \times \mathbb{R}^{3} \mid\|x\|=1,\langle\mathbf{x}, \mathbf{p}\rangle=0, \mathbf{p} \neq 0\right\} .
$$

The manifold $P$ is equipped with the symplectic form which is the restriction of the canonical symplectic form $\sum_{i=1}^{3} \mathrm{~d} x_{i} \wedge \mathrm{d} p_{i}$ on $T^{*} \mathbb{R}^{3}=\mathbb{R}^{3} \times \mathbb{R}^{3}$ to $P$.

Lemma III.4. The manifold $P$ in (22) is diffeomorphic to $\mathrm{SO}(3) \times(0, \infty)$.

c) Symplectic Reduction: We will find an $S^{1}$ symmetry in our time-optimal control problem and perform a symplectic reduction of the problem by this symmetry. Refer to [1] for the symplectic reduction theory and to [2] for its application to optimal control.

Define an $S^{1}$-action on $P$ in (22) as follows:

$$
e^{i \theta} \cdot(\mathbf{x}, \mathbf{p})=\left(R_{\theta}^{\mathbf{x} \times \mathbf{p}} \cdot \mathbf{x}, R_{\theta}^{\mathbf{x} \times \mathbf{p}} \cdot \mathbf{p}\right)
$$

for $e^{i \theta} \in S^{1}$, and $(\mathbf{x}, \mathbf{p}) \in P$ where $R_{\theta}^{\mathbf{x} \times \mathbf{p}}$ is the $3 \times 3$ rotational matrix by angle $\theta$ with the axis in the direction of $\mathbf{x} \times \mathbf{p}$. One can check that this action is symplectic and its momentum map $J: P \rightarrow \mathbb{R}$ is given by

$$
J(\mathbf{x}, \mathbf{p})=\|\mathbf{x} \times \mathbf{p}\| .
$$

Refer to [1], [10] for this computation. Notice that every (nonzero) value of $J$ on $P$ is a regular value. From the vector identity $\|\mathbf{x} \times \mathbf{p}\|^{2}=\|\mathbf{x}\|^{2}\|\mathbf{p}\|^{2}-|\langle\mathbf{x}, \mathbf{p}\rangle|^{2}$ and Lemma III.4, it follows that each level set $J^{-1}(\mu)$ with $\mu \in(0, \infty)=\operatorname{Im} J$ is diffeomorphic to $\mathrm{SO}(3)$. For our purpose of solving the time-optimal control problem (1)-(3), it suffices to consider the case $\mu=1$ since other cases are diffeomorphic to this case.

Lemma III.5. In this symplectic reduction picture, the canonical projection $\pi: J^{-1}(1) \rightarrow J^{-1}(1) / S^{1}$ is isomorphic to $\pi: \mathrm{SO}(3) \subset P \rightarrow S^{2} \subset \mathbb{R}^{3}$ where

$$
\pi:(\mathbf{x}, \mathbf{p}) \mapsto \mathbf{L}=\mathbf{x} \times \mathbf{p} \in \mathbb{R}^{3} .
$$

The symplectic structure on $S^{2}$ comes from the canonical Poisson structure on $\mathbb{R}^{3}$. Moreover, the Hamiltonian in (15) is invariant under the $S^{1}$-action in (23), and its reduced Hamiltonian on $S^{2} \times[-1,1]^{2}$ is given by

$$
H\left(\mathbf{L} ; \omega_{1}, \omega_{3}\right)=\omega_{1} L_{1}+\omega_{3} L_{3} .
$$

In this symplectic reduction, we regard $\omega_{1}$ and $\omega_{3}$ as parameters.

Along each optimal trajectory, the function $M$ in (18) and (19) satisfies

$$
\left|L_{1}(t)\right|+\left|L_{3}(t)\right|=\left|L_{1}(0)\right|+\left|L_{3}(0)\right|
$$

for $t \in\left[0, T_{\min }\right]$. The reduced dynamics of the Hamiltonian $H$ on $S^{2} \subset \mathbb{R}^{3}$ is given by

$$
\dot{\mathbf{L}}=A\left(\omega_{1}, \omega_{3}\right) \mathbf{L}
$$

where $A\left(\omega_{1}, \omega_{3}\right)$ is given in (11). The dynamics in (26) can be derived by $\dot{L}_{i}=\left\{L_{i}, H\right\}, i=1,2,3$. By the definition of $\mathbf{L}$, we have

$$
\langle\mathbf{x}(t), \mathbf{L}(t)\rangle=0, \forall t .
$$

Notice that the optimal control in (17) depends on the reduced dynamics as follows:

$$
\omega_{1}(t)=\operatorname{sign}\left(L_{1}(t)\right), \quad \omega_{3}(t)=\operatorname{sign}\left(L_{3}(t)\right) .
$$


d) Switching Law: We now study the switching law in (28). Recall that we seek an optimal trajectory satisfying (14). For convenience, we visualize both $\mathbf{x}$ and $\mathbf{L}$ in the same $\mathbb{R}^{3}$-space. It is useful to notice from (10) and (26) that if $\left(\omega_{1}, \omega_{3}\right)$ is constant in a time interval $[a, b]$, then for $t \in[a, b]$

$$
\mathbf{x}(t)=e^{t A\left(\omega_{1}, \omega_{3}\right)} \mathbf{x}(a), \quad \mathbf{L}(t)=e^{t A\left(\omega_{1}, \omega_{3}\right)} \mathbf{L}(a)
$$

where

$$
\begin{aligned}
& e^{t A\left(\omega_{1}, \omega_{3}\right)} \\
& =\left[\begin{array}{ccc}
\frac{\left(\omega_{1}\right)^{2}+\left(\omega_{3}\right)^{2} \cos (\omega t)}{\omega^{2}} & -\frac{\omega_{3} \sin (\omega t)}{\omega} & \frac{\omega_{1} \omega_{3}(1-\cos (\omega t))}{\omega^{2}} \\
\frac{\omega_{3} \sin (\omega t)}{\omega} & \cos (\omega t) & -\frac{\omega_{1} \sin (\omega t)}{\omega^{2}} \\
\frac{\omega_{1} \omega_{3}(1-\cos (\omega t))}{\omega^{2}} & \frac{\omega_{1} \sin (\omega t)}{\omega} & \frac{\left(\omega_{3}\right)^{2}+\left(\omega_{1}\right)^{2} \cos (\omega t)}{\omega^{2}}
\end{array}\right]
\end{aligned}
$$

with $\omega=\sqrt{\left(\omega_{1}\right)^{2}+\left(\omega_{3}\right)^{2}}$.

From (27) and (2), it follows that $\mathbf{L}(0)$ lies on the unit circle in the $x_{2}-x_{3}$ plane and $\mathbf{L}\left(T_{\min }\right)$ lies on the unit circle in the $x_{1}-x_{2}$ plane. We now consider the three cases:

$$
\mathbf{L}(0) \in R_{1}, \quad \mathbf{L}(0) \in R_{2}, \quad \text { and } \quad \mathbf{L}(0) \notin R_{1} \cup R_{2},
$$

where

$$
R_{1}=\{(0, \pm 1,0)\}, \quad R_{2}=\{(0,0, \pm 1)\} .
$$

First, we consider the case of $\mathbf{L}(0) \in R_{1}=\{(0, \pm 1,0)\}$. Suppose there exists an optimal trajectory with $\mathbf{L}(0)=$ $(0,1,0)$ (the case of $\mathbf{L}(0)=(0,-1,0)$ can be handled similarly). By (25), $\left|L_{1}(t)\right|+\left|L_{3}(t)\right|=0$ for all $t$, thus

$$
\mathbf{L}(t)=(0,1,0) \forall t \text {. }
$$

due to continuity of $\mathbf{L}(t)=\mathbf{x}(t) \times \mathbf{p}(t)$ in $t$. As the $\mathbf{x}-$ trajectory moves from $(1,0,0)$ to $(0,0,1)$, it is impossible to have $\left(\omega_{1}(t), \omega_{3}(t)\right)=(0,0)$ almost everywhere. Hence, there exists $t_{1}<T_{\min }$ such that

$$
\int_{0}^{t_{1}} \omega_{1}(s) d s \neq 0 \text { or } \int_{0}^{t_{1}} \omega_{3}(s) d s \neq 0 .
$$

Let us consider the former case since the latter can be handled similarly. We have

$$
L_{3}\left(t_{1}\right)=\int_{0}^{t_{1}} \omega_{1}(s) L_{2}(s) d s=\int_{0}^{t_{1}} \omega_{1}(s) d s \neq 0
$$

which is a contradiction to (31). Hence, $\mathbf{L}(0) \in R_{1}=$ $\{(0, \pm 1,0)\}$ cannot generate optimal trajectories. (25),

We now consider the second case, $\mathbf{L}(0)=(0,0,1)$. By

$$
\left|L_{1}(t)\right|+\left|L_{3}(t)\right|=1 \quad \forall t .
$$

By (32) and the orthogonality of $\mathbf{L}\left(T_{\min }\right)$ to $\mathbf{x}\left(T_{\min }\right)=$ $(0,0,1)$, it is necessary that $\mathbf{L}\left(T_{\min }\right)=(1,0,0)$ or $(-1,0,0)$. Take an arbitrary positive $\delta \leq T_{\min }$ such that

$$
L_{3}(t)>0 \quad \forall t \in[0, \delta],
$$

which is possible by the continuity of $L_{3}(t)$. If there is $\bar{t} \in$ $(0, \delta]$ such that $L_{1}(\bar{t})=0$, then $\mathbf{L}(\bar{t})=(0,0,1)$ by (32) and (33). This implies that point $(0,0,1)=\mathbf{L}(\bar{t})$ is transferred to point $\pm(1,0,0)=\mathbf{L}\left(T_{\min }\right)$ with time cost $\left(T_{\min }-\bar{t}\right)$.
It follows that the minimum time cost for $\mathbf{x}(t)$ should be at most $\left(T_{\min }-\bar{t}\right)$ by Lemma III.3, which contradicts the definition of $T_{\min }$. Therefore, $L_{1}(t)$ never vanishes on $(0, \delta]$. Hence, either $L_{1}(t)<0$ for all $t \in(0, \delta]$ or $L_{1}(t)>0$ for all $t \in(0, \delta]$.

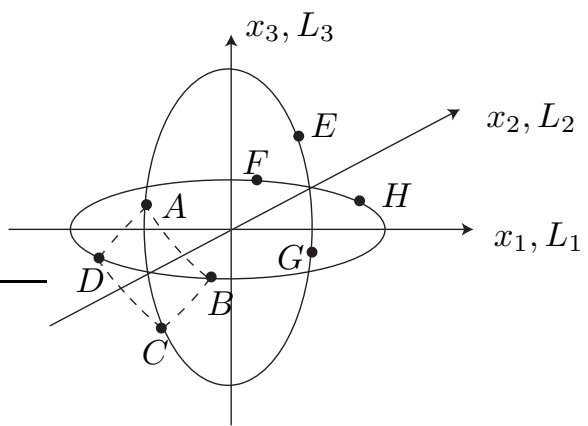

(a)

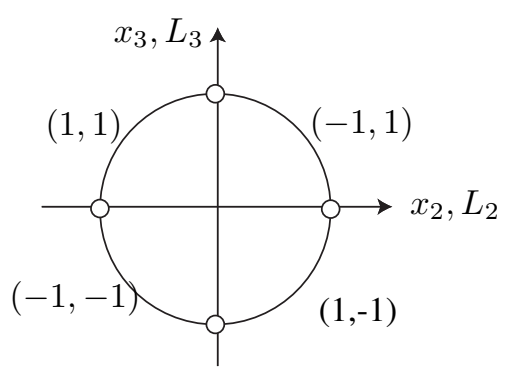

(b)

Fig. 1. (a) The initial value $\mathbf{L}(0)$ must lie on the unit circle on the $x_{2}-x_{3}$ plane. The final value $\mathbf{L}\left(T_{\min }\right)$ must lie on the unit circle on the $x_{1}-x_{2}$ plane. (b) The initial value of the control $\left(\omega_{1}(0), \omega_{3}(0)\right)$ corresponding to $\mathbf{L}(0)$ on the unit circle on the $x_{2}-x_{3}$ plane minus $\{(0,0, \pm 1),(0, \pm 1,0)\}$.

Suppose that $L_{1}(t)<0$ for all $t \in(0, \delta]$. Then, $\left(\omega_{1}(t), \omega_{3}(t)\right)=(-1,1)$ on $(0, \delta]$. Using (29) and (30), we get $x_{3}(t)=-\frac{1}{2}(1-\cos (\sqrt{2} t))$ for $t \in(0, \delta]$. For a sufficiently small $t$, we get $x_{3}(t)<0$. Hence, $x_{3}([0, \delta])$ is not contained in $\overline{\mathcal{O}}_{1}$, which contradicts (14). Therefore, $L_{1}(t)>0$ for all $t \in(0, \delta]$ where $\delta$ is an arbitrary positive number less than or equal to $T_{\min }$ such that (33) holds. Simple integration of (10) and (26) with (29) and (30) yields the following: for all $t \in\left(0, \frac{\pi}{\sqrt{2}}\right)$

$$
\begin{aligned}
\omega_{1}(t) & =1, \quad \omega_{3}(t)=1, \\
\mathbf{x}(t) & =\left(\frac{1+\cos (\sqrt{2} t)}{2}, \frac{\sin (\sqrt{2} t)}{\sqrt{2}}, \frac{1-\cos (\sqrt{2} t)}{2}\right) \\
& \in \operatorname{int}\left(\overline{\mathcal{O}}_{1}\right), \\
\mathbf{L}(t) & =\left(\frac{1-\cos (\sqrt{2} t)}{2},-\frac{\sin (\sqrt{2} t)}{\sqrt{2}}, \frac{1+\cos (\sqrt{2} t)}{2}\right),
\end{aligned}
$$

where in particular, $L_{1}(t)>0$ and $L_{3}(t)>0$. It is easy to see that

$$
\mathbf{x}\left(\frac{\pi}{\sqrt{2}}\right)=(0,0,1), \quad \mathbf{L}\left(\frac{\pi}{\sqrt{2}}\right)=(1,0,0) .
$$


Thus, the trajectory in (34) is a candidate for an optimal trajectory with time cost $T_{\min }=\frac{\pi}{\sqrt{2}}$.

Next, we consider the case $\mathbf{L}(0)=(0,0,-1)$. By the continuity of $\mathbf{L}(t)$, there is $0<\delta<\min \left\{T_{\min }, \frac{1}{100}\right\}$ such that $L_{3}(t)<0$ and $\omega_{3}(t)=\operatorname{sign}\left(L_{3}(t)\right)=-1$ on $[0, \delta]$. Using (29) and (30), we get $x_{2}(t)=-\frac{1}{\omega} \sin (\omega t)<0$ on $(0, \delta]$. Hence, $\mathbf{x}((0, \delta]) \cap \overline{\mathcal{O}}_{1}=\emptyset$, which contradicts the assumption in (14). Thus, we exclude the case $\mathbf{L}(0)=$ $(0,0,-1)$.

Lastly, we consider the case where $\mathbf{L}(0) \notin R_{1} \cup R_{2}$. The unit circle minus $R_{1} \cup R_{2}$ in the $x_{2}-x_{3}$ plane consists of four open arcs; see Figure 1.(b). It is not hard to see that the initial value of optimal control $\left(\omega_{1}, \omega_{3}\right)$ should be given as in Figure 1.(b) depending on the initial value $\left(L_{2}(0), L_{3}(0)\right)$. For example, suppose that $\left(L_{2}(0), L_{3}(0)\right)=A$ in Figure 1.(a), i.e., $L_{1}(0)=0, L_{2}(0)<0, L_{3}(0)>0$. Then, there is $t_{1}>0$ such that $L_{2}(t)<0, L_{3}(t)>0$ on $\left[0, t_{1}\right]$. It follows that $L_{1}(t)=\int_{0}^{t}-\omega_{3}(s) L_{2}(s) d s=\int_{0}^{t}-L_{2}(s) d s>0$ for all $t \in\left(0, t_{1}\right]$. Hence, $\omega_{1}(t)=1$ for all $t \in\left(0, t_{1}\right]$. Hence, we may set $\omega_{1}(0)=1$ since $t=0$ is a measure-zero set. This explains the choice $\left(\omega_{1}(0), \omega_{3}(0)\right)=(1,1)$ in Figure 1.(b). The argument made so far also implies that $\mathbf{L}(t)$ starts to enter the first octant of $\mathbb{R}^{3}$ and remains there with $\left(\omega_{1}(t), \omega_{3}(t)\right)=(1,1)$ until it hits the switching plane $L_{3}=0$. The switching order is summarized in Figure 2 . Recall from (25) that $\mathbf{L}(t)$ on the unit sphere satisfies

$$
\left|L_{1}(t)\right|+\left|L_{3}(t)\right|=\left|L_{1}(0)\right|+\left|L_{3}(0)\right|>0 .
$$

It is straightforward to check that switching is periodic by symmetry, which also can be seen directly from the dotted line in Figure 1.(a).

We claim that $\mathbf{L}(0)$ lies on the open arc in the second quadrant of the $x_{2}-x_{3}$ plane. Suppose that $\mathbf{L}(0)$ lies on the open arc in the first quadrant of the $x_{2}-x_{3}$ plane such as point $E$ in Figure 1.(a). Then, there is a sufficiently small positive $\epsilon<\min \left\{T_{\min }, \frac{1}{100}\right\}$ such that $\left(\omega_{1}(t), \omega_{3}(t)\right)=(-1,1)$ for $t \in[0, \epsilon]$, which by (30) implies $x_{3}(t)=-\frac{(1-\cos (\sqrt{2} t))}{2}<0$ on $(0, \epsilon]$. Therefore, $\mathbf{x}((0, \epsilon]) \cap \overline{\mathcal{O}}_{1}=\emptyset$, which contradicts the assumption in (14). Hence, we exclude this case. In a similar manner, we can exclude the case of $\mathbf{L}(0)$ being on the other two open arcs in the third and fourth quadrants of the $x_{2}-x_{3}$ plane. Therefore, $\mathbf{L}(0)$ must lie on the open arc in the second quadrant of the $x_{2}-x_{3}$ plane.

Let $A=\mathbf{L}(0)$ as in Figure 1.(a). Since $\mathbf{L}\left(T_{\min }\right)$ should be orthogonal to $\mathbf{x}\left(T_{\min }\right)=(0,0,1), \mathbf{L}\left(T_{\min }\right)$ must lie in the plane $L_{3}=0$. Hence, $\mathbf{L}\left(T_{\min }\right)$ must be either point $B$ or point $D$ in Figures 1.(a) and 2. We claim that $\mathbf{L}\left(T_{\min }\right)=B$, that is, $\mathbf{L}\left(T_{\min }\right) \neq D$. Suppose that $\mathbf{L}\left(T_{\min }\right)=D$. Then, according to the scheme in Figure 2, the middle part of the corresponding trajectory $\mathbf{x}(t)$ is in the middle of the time interval on which $\left(\omega_{1}, \omega_{3}\right)= \pm(1,-1)$, so the trajectory remains on plane $\Pi=\left\{x_{1}=x_{3}\right\}$ for a time interval of non-zero length since $\mathbf{x}\left(\frac{T_{\min }}{2}\right) \in \Pi$, which can be easily checked using (29) and (30). Hence, by the third statement in Lemma III.2, it cannot be an optimal trajectory. From this observation, we arrive at:
Lemma III.6. If there is an optimal trajectory with $\mathbf{L}(0)=$ $A$ in the open arc in the second quadrant of the $x_{2}-x_{3}$ plane, then $\mathbf{L}\left(T_{\min }\right)=B$. Consequently, the number of switchings is $0,4,8, \ldots$.
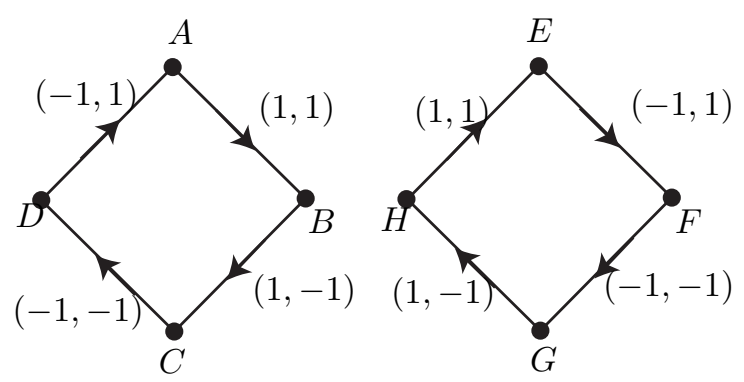

Fig. 2. The switching scheme of the extremal control $\left(\omega_{1}(t), \omega_{3}(t)\right)$ where the points $A, B, C, D, E, F, G$ and $H$ correspond to those in Figure 1.(a).

We now claim that the number of switchings is 0 . Suppose that there is an optimal trajectory with $\mathbf{L}(0)=A$ with the number of switchings greater than or equal to 4 . Let $T_{\mathrm{s}}$ be the switching period. It follows that $T_{\min }>2 T_{\mathrm{s}}$. Since we have found a trajectory in (34) with time cost $\frac{\pi}{\sqrt{2}}$, we have $2 T_{\mathrm{s}}<T_{\min } \leq \frac{\pi}{\sqrt{2}}$. On $\left[0,2 T_{\mathrm{s}}\right]$ the control law is given by

$$
\left(\omega_{1}(t), \omega_{3}(t)\right)= \begin{cases}(1,1) & \text { for } 0 \leq t \leq T_{\mathrm{s}} \\ (1,-1) & \text { for } T_{\mathrm{s}}<t \leq 2 T_{\mathrm{s}}\end{cases}
$$

and by (29) and (30), we get $\mathrm{x}\left(2 T_{\mathrm{s}}\right)=$ $e^{T_{\mathrm{s}} A(1,-1)} \cdot e^{T_{\mathrm{s}} A(1,1)} \mathbf{x}(0)$, which implies $x_{2}\left(2 T_{\mathrm{s}}\right)=$ $\frac{1}{\sqrt{2}} \sin \left(\sqrt{2} T_{\mathrm{s}}\right)\left(\cos \left(\sqrt{2} T_{\mathrm{s}}\right)-1\right)<0$ since $0<\sqrt{2} T_{s}<\frac{\pi}{2}$. We exclude this trajectory since it is not contained in $\overline{\mathcal{O}}_{1}$ as assumed in (14). Hence, the only possible optimal control would be $\left(\omega_{1}, \omega_{3}\right)=(1,1)$ without switchings, which we have already studied and have found the trajectory in (34). We have so far proved the following:

Claim III.7. There is only one optimal trajectory contained in the first closed octant $\overline{\mathcal{O}}_{1}$. It is given by

$$
\mathbf{x}(t)=\left(\frac{1+\cos (\sqrt{2} t)}{2}, \frac{\sin (\sqrt{2} t)}{\sqrt{2}}, \frac{1-\cos (\sqrt{2} t)}{2}\right)
$$

with the control $\left(\omega_{1}(t), \omega_{3}(t)\right)=(1,1)$ and the time cost $T_{\min }=\frac{\pi}{\sqrt{2}}$. Moreover, it is $g_{4}$-invariant.

Theorem III.8. There are only two optimal trajectories, and the minimum time cost is $\frac{\pi}{\sqrt{2}}$. One is given in (35) with the control $\left(\omega_{1}, \omega_{3}\right)=(1,1)$ and the other is given by

$$
g_{2}(\mathbf{x}(t))=\left(\frac{1+\cos (\sqrt{2} t)}{2},-\frac{\sin (\sqrt{2} t)}{\sqrt{2}}, \frac{1-\cos (\sqrt{2} t)}{2}\right)
$$

with the control $\left(\omega_{1}, \omega_{3}\right)=(-1,-1)$.

\section{Generalization}

We now show to what extent the techniques used for the 3 -level system can be applied to the general $n$-level system in (4)-(6), and leave some comments for the readers. The dynamics have symmetry $G=\left\langle g_{i} \mid i=1, \ldots, n\right\rangle$ where 
each $g_{i}$ is the reflection in the plane $\left\{x_{i}=0\right\}$. Hence, there is an optimal trajectory in $\left\{x_{i} \geq 0, i=1, \ldots, n\right\}$.

There is an additional discrete symmetry. For $n=2 k$, if there is a trajectory $\mathbf{x}(t), 0 \leq t \leq T$, connecting the initial point to the final point, then one can construct a trajectory $\mathbf{y}(t), 0 \leq t \leq T$, connecting the initial point to the final point such that $\mathbf{y}(t)=S(\mathbf{x}(T-t))$ where $S=(1,2 k)(2,2 k-1) \cdots(k, k+1)$ is a permutation on the index set $\{1,2, \ldots, 2 k\}$. For $n=2 k+1$, the same holds with $S=(1,2 k+1)(2,2 k) \cdots(k-1, k+1)$. However, the existence of an optimal trajectory which is invariant under $S$ is in general unknown for $n>3$.

Following the Pontryagin Maximum Principle, we first set up the Hamiltonian

$$
H(\mathbf{x}, \mathbf{p} ; u)=u_{1}\left(x_{1} p_{2}-x_{2} p_{1}\right)+\cdots+u_{n-1}\left(x_{n-1} p_{n}-x_{n} p_{n-1}\right)
$$

where the covector $\mathbf{p}$ obeys the same dynamics as those in (4). The optimal control satisfies

$$
u_{i}(t)=\operatorname{sign}\left(x_{i}(t) p_{i+1}(t)-x_{i+1}(t) p_{i}(t)\right),
$$

and along each optimal trajectory

$$
M(t)=\sum_{i=1}^{n-1}\left|x_{i}(t) p_{i+1}(t)-x_{i+1}(t) p_{i}(t)\right|=\text { constant. }
$$

By Corollary II. 2 and the fact that $\|\mathbf{x}(t)\|=1$, we have the transversality condition

$$
p_{1}(0)=0 \text {. }
$$

Since $\mathbf{x}(t)$ is perpendicular to $\mathbf{p}(t)$ for all $t$, we may regard the Hamiltonian $H$ as a function defined on

$$
P=\left\{(\mathbf{x}, \mathbf{p}) \in \mathbb{R}^{n} \times \mathbb{R}^{n} \mid\|\mathbf{x}\|=1,\langle\mathbf{x}, \mathbf{p}\rangle=0, \mathbf{p} \neq 0\right\}
$$

where $\mathbf{p} \neq 0$ comes from the Pontryagin Maximum Principle. Here, the manifold $P$ has the symplec structure induced from the canonical form $\Omega=\sum_{i=1}^{n} d x_{i} \wedge d p_{i}$. It is easy to see that $P$ is diffeomorphic to $T_{1} S^{n-1} \times(0, \infty)$ where $T_{1} S^{n-1}$ is the unit tangent space of the $(n-1)$-sphere.

We now detect continuous symmetry in the Hamiltonian and perform symplectic reduction. Consider the function

$$
J(\mathbf{x}, \mathbf{p})=\|\mathbf{x}\|^{2}\|\mathbf{p}\|^{2}-|\langle\mathbf{x}, \mathbf{p}\rangle|^{2} .
$$

We denote the Hamiltonian vector field of $J$ by $X_{J}$. On the manifold $P$, the vector field $X_{J}$ is given by

$$
\begin{aligned}
\left.X_{J}\right|_{P} & =\left.\left(2\|\mathbf{x}\|^{2} \mathbf{p}+2\langle\mathbf{x}, \mathbf{p}\rangle \mathbf{x},-2\|\mathbf{p}\|^{2} \mathbf{x}-2\langle\mathbf{x}, \mathbf{p}\rangle \mathbf{p}\right)\right|_{P} \\
& =\left(2 \mathbf{p},-2\|\mathbf{p}\|^{2} \mathbf{x}\right) .
\end{aligned}
$$

One can verify that $X_{J}$ is tangent to $P$ at each point of $P$, so $P$ is an invariant manifold of $X_{J}$. The flow $\varphi_{t}^{X_{J}}$ of $X_{J}$ on $P$ is given by

$$
\varphi_{t}^{X_{J}}(\mathbf{x}, \mathbf{p})=\left[\begin{array}{cc}
\cos (2 \mu t) I_{n} & \frac{1}{\mu} \sin (2 \mu t) I_{n} \\
-\mu \sin (2 \mu t) I_{n} & \cos (2 \mu t) I_{n}
\end{array}\right] \cdot\left[\begin{array}{l}
\mathbf{x} \\
\mathbf{p}
\end{array}\right]
$$

where $I_{n}$ is the $n \times n$ identity matrix and $\mu=\|\mathbf{p}\|$. Here, it is understood that $\|\mathbf{p}\|$ is constant along the flow of $X_{J}$ on $P$, which can be easily verified by computing $X_{J} \cdot\|\mathbf{p}\|=0$ on $P$. Since each flow $\varphi_{t}^{X_{J}}(\mathbf{x}, \mathbf{p})$ is periodic with period $\frac{\pi}{\|\mathbf{p}\|}$, we can define a $S^{1}$-action on $P$ by

$$
e^{i \theta} \cdot(\mathbf{x}, \mathbf{p})=\varphi_{\frac{\theta}{2\|\mathbf{p}\|}}^{X_{J}}(\mathbf{x}, \mathbf{p}), \quad \theta \in[0,2 \pi] .
$$

This action is symplectic since it is the Hamiltonian flow $\varphi_{t}^{X_{J}}$. Since $\{H, J\}_{\mathbb{R}^{4} \times \mathbb{R}^{4}}=0, H$ is constant under this action.

For the purpose of finding optimal trajectories, we fix the level of the momentum map $J$ at $\mu=1$ since other level sets of $J$ on $P$ are diffeomorphic to $J^{-1}(1)$. We note that

$$
\begin{aligned}
J^{-1}(1) & =\left\{(\mathbf{x}, \mathbf{p}) \in \mathbb{R}^{n} \times \mathbb{R}^{n} \mid\|\mathbf{x}\|=\|\mathbf{p}\|=1,\langle\mathbf{x}, \mathbf{p}\rangle=0\right\} \\
& =T_{1} S^{n-1} .
\end{aligned}
$$

By the symplectic reduction theory, there is a projection $\pi$ : $J^{-1}(1) \rightarrow J^{-1}(1) / S^{1}$ and a reduced Hamiltonian $h(r ; u)$ on $J^{-1}(1) / S^{1}$ such that

$$
H(\mathbf{x}, \mathbf{p} ; u)=h(r ; u), \quad r=\pi(\mathbf{x}, \mathbf{p})
$$

where the control $u=\left(u_{1}, \cdots, u_{n-1}\right)$ is regarded as a parameter in this reduction process. Moreover, there is a symplectic form $\omega$ on $J^{-1}(1) / S^{1}$ such that $\left.\Omega\right|_{J^{-1}(1)}=\pi^{*} \omega$.

By the Pontryagin Maximum Principle, optimal control maximizes $H(\mathbf{x}, \mathbf{p}, u)$, but through the symplectic reduction it is equivalent to maximizing the reduced Hamiltonian $h(r, u)$ for $r \in J^{-1}(1) / S^{1}$. Thus, the switching of $u_{i}$ in (36) depends on the Hamiltonian dynamics of $h(r ; u)$ on the $(2 n-4)$ dimensional space $J^{-1}(1) / S^{1}$, rather than on the $(2 n-3)$ dimensional space $J^{-1}(1)$, which would be hard to detect without symplectic reduction. The reduction process will be illustrated with the 4-level system in a forthcoming paper.

\section{REFERENCES}

[1] R. Abraham and J.E. Marsden, Foundations of mechanics, Addison Wesley Publishing Company, 1978.

[2] G. Blankenstein and A. van der Schaft, Optimal control and implicit Hamiltonian systems in Nonlinear Control in the Year 2000, Vol 1 Lecture Notes in Control and Information Sciences, 258:185-205, 2001

[3] U. Boscain, T. Chambrion and G. Charlot, Nonisotropic 3-level quantum systems: complete solutions for minimum time and minimal energy, Discrete and Continuous Dynamical Systems-B, 5(4): 957-990, 2005.

[4] U. Boscain, T. Chambrion and J-P. Gauthier, Optimal control on a $n$ level quantum system Lagrangian and Hamiltonian methods in Nonlinear Control 2003 Elsevier (IFAC Proceedings Volumes), Sevilla, Spain, pp. 151-156, 2003.

[5] U. Boscain, G. Charlot, Resonance of minimizers for $n$-level quantum systems with an arbitrary cost, ESAIM: Control, Optimisation and Calculus of Variations (COCV), 10: 593-614, 2004.

[6] U. Boscain and P. Mason, Time minimal trajectories for a spin 1/2 particle in a magnetic field , Journal of Mathematical Physics, 47(6): $06201,2006$.

[7] V. Jurdjevic, Geometric control theory, Cambridge University Press, 1997.

[8] E.B. Lee and L. Markus, Foundations of optimal control theory, New York, John Wiley \& Sons, Inc., 1967.

[9] L.S. Pontryagin, V.G. Boltyanskii, R.V. Gamkrelidze, and E.F. Mishchenko, The mathematical theory of optimal processes, New York, John Wiley \& Sons, Inc., 1962.

[10] J.E. Marsden and T.S. Ratiu, Introduction to mechanics and symmetry. Springer, 1999.

[11] P. Rouchon, Flatness based trajectory generation of quantum systems, Proc. NOLCOS, Stuttgart, Germany, 2004. 